\title{
Zur Kenntnis der Plasteine.
}

Von

J. Herrmann und A. Chain.

(Aus dem physiologischen Institut in Odessa.)

(Der Redaktion zugegangen am 2. März 1912.)

Bekanntlich ist von vielen Autoren festgestellt worden, daß bei der Präzipitinreaktion nur Eiweißstoffe als Antigene fungieren können. Außerdem ist durch die Arbeiten von Obermeyer und Pick gezeigt, daß, wenn ein zu einem bestimmten Eiweißstoff gehöriges Präzipitin nicht nur mit seinem Präzipitogen, sondern auch mit einem anderen Eiweißstoff reagiert, diese Reaktion als ein Beweis der Anwesenheit identischer Atomkomplexe in diesen Eiweißstoffen betrachtet wird. Somit kann uns die Präzipitinreaktion als ein Mittel dienen, um die Eiweißnatur eines Stoffes festzustellen, und ebenso um eine gewisse Analogie im molekularen Bau der gegebenen Eiweißstoffe $\mathrm{zu}$ erweisen.

Demgemäß haben wir nach dem Vorschlage unseres hochgeehrten Lehrers, Herrn Prof. Saw jaloff, die Präzipitinreaktion zur Untersuchung der Eiweißnatur und der Identität der Plasteine benutzt. In unseren Versuchen haben wir durch Vorbehandlung der Kaninchen mit neutralen Lösungen von Plastein, welches aus Witte-Pepton dargestellt war, Antisera gewonnen. Die Versuche haben uns gezeigt, daß die gewonnenen Antisera nicht nur mit ihrem Präzipitogen, sondern auch mit anderen Plasteinen, die aus verschiedenen Eiweißstoffen, z. B. aus Edestin, Serumalbumin, Eieralbumin, Mandelglobulin, dargestellt waren, reagieren. Was die Eiweißstoffe, aus denen unsere Plasteine dargestellt waren, betrifft - wir haben in dieser Hinsicht WittePepton und Casein geprüft -, so reagieren sie mit unserem Antiserum nicht.

Unsere Versuche - meinen wir - bestätigen die Eiweißnatur der Plasteine; außerdem schließen wir auf Grund unserer Reaktionen, daß die Moleküle verschiedener Plasteine, da sie sich gegenüber der Präzipitinreaktion gleichartig verhalten, eine gewisse Analogie in ihrem Bau besitzen müssen. 